\title{
Local Cohomology and Superselection Structure
}

\author{
John E. Roberts \\ Centre de Physique Théorique, C.N.R.S., F-13274 Marseille Cedex 2, France
}

\begin{abstract}
If $A^{\mu}$ is a vector field satisfying $\partial^{\mu} A^{v}-\partial^{v} A^{\mu}=0$ can one find a scalar field $\phi$ such that $A^{\mu}=\dot{\partial}^{\mu} \phi$ ? A novel quantum analogue of this classical problem incorporating locality is introduced and is shown to generate those superselection sectors whose charge can be strictly localized. In a 2-dimensional space-time there are further possibilities; in particular, soliton sectors can be generated by this procedure.
\end{abstract}

\section{Introduction}

The possibility of describing superselection structure in elementary particle physics in terms of observable quantities was first pointed out by Haag and Kastler [1]. Their idea was that the charge quantum numbers should appear as labels for those inequivalent irreducible representations of the observable algebra whose vector states are relevant to elementary particle physics. The first attempt to give concrete shape to these ideas was undertaken by Borchers [2]. He postulated that the relevant representations were those which are "strongly local equivalent" to the vacuum representation and showed how the unobservable fields could be recovered as the intertwining operators which realize this equivalence. However his postulates, already suspect because his analysis apparently ruled out the possibility of parastatistics, were shown in [3] to be violated if the superselection sectors are generated, as in conventional field theory, by a principle of gauge invariance of the first kind. Nevertheless a slight modification of the terms "strong local equivalence" allowed Borchers' results for systems obeying ordinary Bose and Fermi statistics to be recovered and extended [4].

The systematic treatment of superselection structure in $[5,6]$ classifies the particle statistics compatible with locality and analyses the operations of charge addition and charge conjugation. On its own terms, this analysis is rather complete; it is true that it has not yet proved possible to show that the superselection structure may always be described as the representation theory of some compact gauge group. However, even if such a result holds, it would add little to our 
understanding of the structure. If it does not hold, it is conceivable, but perhaps unlikely, that it would open new, interesting possibilities for the phenomenological classification of elementary particles.

The real weakness of the present analysis lies elsewhere: it is quite possible that the most interesting quantum field theory models do not fall within the scope of the analysis. This is certainly the case with quantum electrodynamics because, as pointed out in [5], sectors with non-zero electric charge do not satisfy the selection criterion used there. Furthermore as gauge groups of the first kind are so intimately related to superselection structure, the question naturally arises as to whether this is also the case with gauge groups of the second kind. Admittedly, the naive answer is that gauge invariance of the second kind does not generate superselection sectors but serves instead to rule out unphysical states. However, in the absence of a deeper understanding of its intrinsic role, this conclusion is suspect.

The approach advocated here has the great advantage of promising to resolve these problems as well as shedding light on other structural features of quantum field theory. Instead of studying representations that satisfy some selection criterion as in [5], the superselection structure will be deduced as part of the analysis of the "local cohomology" of the observables. The "local cohomology" may be thought of as the quantum analogue of the cohomology of differential forms. Here we shall only be using the local 1-cohomology of the observables. We recall that in the cohomology of differential forms, a closed 1 -form corresponds to a vector field $A^{\mu}(x)$ whose curl vanishes

$$
\partial^{\mu} A^{v}(x)-\partial^{v} A^{\mu}(x)=0
$$

and it is exact if there is a scalar field $\phi(x)$ such that

$$
A^{\mu}(x)=\partial^{\mu} \phi(x) \text {. }
$$

These equations may be looked at in integrated form. If $b$ is any path, i.e. a smooth mapping of the unit interval $[0,1]$ into Minkowski space, we may consider the path integral

$$
A(b)=\int_{0}^{1} A^{\mu}(b(s)) \frac{d b_{\mu}(s)}{d s} d s .
$$

Equation (1.1) means that the integral round a closed path bounding a 2-dimensional surface is zero and (1.2) that

$$
A(b)=\phi(b(1))-\phi(b(0)) \text {. }
$$

The most obvious way of producing a quantum analogue for the equations is to require that $A^{\mu}(x)$ be a local quantum vector field satisfying (1.1) and to ask whether there is a local quantum scalar field $\phi(x)$ satisfying (1.2). The really important new ingredient here is the locality requirement. This question, which arises naturally in discussing certain models, has been treated by Pohlmeyer [7]. He shows, among other things, that, in a covariant theory with a mass gap in more than two space-time dimensions, it is always possible to find such a field $\phi(x)$. From the point of view adopted here this is a negative result, analogous to the Goldstone theorem, showing that (1.1) cannot generate superselection sectors 
under the above conditions just as the Goldstone theorem shows that a conserved current cannot generate new vacuum sectors in the absence of zero-mass particles.

The more interesting analogue of (1.1) and (1.2) avoids using unbounded operator-valued distributions which, quite apart from being awkward to handle mathematically, have the flaw of making the cohomology classes into real vector spaces. It is based on a notion of "charge transfer" along a path. There are three physically plausible properties of this operation ${ }^{1}$ :

a) invertibility (we can transfer charge back along the same path),

b) charge neutrality (the total charge is unchanged),

c) localizability (the operation should be localized about the path in question).

If we assume as in [5] that the observable algebra satisfies duality in the vacuum sector, i.e. for each double cone $\mathcal{O}$ we have in the vacuum representation

$$
\mathfrak{U}\left(\mathcal{O}^{\prime}\right)^{\prime}=\mathfrak{U}(\mathcal{O}) .
$$

Then the above properties imply that the operation of charge transfer is an inner automorphism of the observable algebra generated by a unitary operator from some $\mathfrak{A}(\mathcal{O})$.

If we transfer charge along a path from a) to b) and then along a path from b) to c) we may plausibly assume that the net result is the same as if charge is transferred along the composed path from a) to c). The really crucial restrictive assumption we make here is that the operation of transferring charge along a path from a) to b) is independent of the path chosen and depends only on the endpoints a) and b). It is here that we exclude the electric charge because, by a plausible extrapolation of Maxwell's equations, the effect of transferring charge around a closed path should be the operation of creating a flux of magnetic field through a surface spanning this path.

The above discussion may be summed up by saying that we assume that the operation of charge transfer is represented by a local 1-cocycle over Minkowski space with values in the group of inner automorphisms of the observable algebra. The precise definition of these terms will be deferred until Section 2 where we show that the resulting local 1 -cohomology is equivalent to the approach to superselection structure described in [5]. However the relation between charge transfer and superselection sectors is quite simple and has already been discussed in [5]. A limiting procedure allows one to transfer charge in from spacelike infinity; the resulting operation is, in general, no longer an inner automorphism of the observable algebra but leads to a new sector and describes the creation of a localized charge.

It is perhaps not surprising that the above results must be modified in a 2-dimensional space-time where one may distinguish a spacelike left from a spacelike right. If one tries to create a charge by transferring charge in from spacelike left infinity, say, the resulting operation is not necessarily localized. The operation is trivial on the spacelike right but on the spacelike left it may correspond to performing an internal symmetry on the system. This internal symmetry is then the result of transferring charge from spacelike left infinity to spacelike right infinity. If, as can happen, this internal symmetry is spontaneously broken, then

1 The term operation here is being used in a technical sense, see [1]. 
one is describing a typical aspect of soliton behaviour and the resulting sectors may be regarded as soliton sections. This forms the subject matter of Section 3.

To understand how the local 1-cohomology comes to be related to internal symmetry, one may recall that, in a 2-dimensional space-time, the dual tensor of a vector field satisfying (1.1) is a conserved current. In fact this aspect of local 1-cohomology, in contrast to its relation to superselection sectors, emerges clearly from the discussion in [7]. Reverting now to the four dimensions of physical space-time, the local 3-cohomology will correspond to the notion of a conserved current, $\partial_{\mu} j^{\mu}=0$. Hence one may plausibly conjecture that soliton behaviour is now associated with the local 3-cohomology. Indeed the higher local cohomologies promise to be an interesting field of study for other reasons as well. Thus the local 3-cohomology in its role of generating a gauge symmetry should couple with the local 1-cohomology to describe the measurement of charge. The local 2-cohomology, exemplified by one of Maxwell's equations $\partial^{\{\lambda} F^{\mu \nu\}}=0$, should lead to a description of theories such as electrodynamics with a gauge group of the second kind.

\section{Local Cohomology}

We suppose given the observable net $\mathcal{O} \rightarrow \mathfrak{U}(\mathcal{O}), \mathcal{O} \in \mathscr{K}$ of von Neumann algebras on the Hilbert space $\mathscr{H}_{0}$ of the vacuum representation. Here $\mathscr{K}$ denotes the set of closed double cones in Minkowski space and we shall use $\mathscr{K}_{0}$ to denote the subset of double cones centred on the origin. We suppose, as usual, that $\mathcal{O}_{1} \subset \mathrm{O}_{2}$ implies $\mathfrak{A}\left(\mathcal{O}_{1}\right) \subset \mathfrak{U}\left(\mathcal{O}_{2}\right)$ and let $\mathfrak{U}$ denote the $C^{*}$-inductive limit of the algebras $\mathfrak{A}(\mathcal{O}), \mathfrak{A}$ is supposed to be irreducible on $\mathscr{H}_{0}$. If $\mathcal{O}^{\prime}$ denotes the spacelike complement of $\mathcal{O}$, then $\mathfrak{A}\left(\mathcal{O}^{\prime}\right)$ denotes the $C^{*}$-subalgebra of $\mathfrak{A}$ generated by $\left\{\mathfrak{U}\left(\mathcal{O}_{1}\right)\right.$; $\left.\mathcal{O}_{1} \subset \mathcal{O}^{\prime}, \mathcal{O}_{1} \in \mathscr{K}\right\}$. As indicated in the introduction, we suppose that $\mathcal{O} \rightarrow \mathfrak{U}(\mathcal{O})$ is not only local but also satisfies the duality condition (1.5).

By a morphism of $\mathfrak{A}$ we mean a linear mapping $\varrho: \mathfrak{U} \rightarrow \mathfrak{U}$ such that $\varrho(A B)=$ $\varrho(A) \varrho(B), \varrho(A)^{*}=\varrho\left(A^{*}\right), A, B \in \mathfrak{U}$ and $\varrho(I)=I$. We let Mor $\mathfrak{A}$ denote the set of morphisms of $\mathfrak{A}$. We say that two morphisms $\varrho_{1}$ and $\varrho_{2}$ are equivalent if there is an inner automorphism $\sigma$ such that $\varrho_{1}=\sigma \varrho_{2}$. If we let $\pi_{0}$ denote the inclusion mapping of $\mathfrak{A}$ into $\mathscr{B}\left(\mathscr{H}_{0}\right)$, the set of bounded linear operators on $\mathscr{H}_{0}$, then $\pi_{0} \circ \varrho$ is again a representation of $\mathfrak{U}$ on the Hilbert space $\mathscr{H}_{0}$. We say that a morphism $\varrho$ is localized in a compact subset $F$ of Minkowski space if for any $\mathcal{O} \in \mathscr{K}, \mathcal{O} \subset F^{\prime}$

$$
\varrho(A)=A, \quad A \in \mathfrak{U}(\mathcal{O}) .
$$

As a consequence of duality, two localized morphisms $\varrho_{1}$ and $\varrho_{2}$ are equivalent if and only if the corresponding representations $\pi_{0}^{\circ} \varrho_{1}$ and $\pi_{0} \circ \varrho_{2}$ are unitarily equivalent. As in [5], we write $\varrho \in \Delta_{t}(\mathcal{O})$ if $\varrho$ is localized in $\mathcal{O}$ and if, given any translate $\mathcal{O}+x$ of $\mathcal{O}$, there is an equivalent morphism $\varrho^{\prime}$ localized in $\mathcal{O}+x$. We set $\Delta_{t}=\bigcup_{\mathcal{O} \in \mathscr{K}} \Delta_{t}(\mathcal{O})$ and refer to the elements of $\Delta_{t}$ as transportable localized morphisms.

In these terms, the basic assumption in [5] may be expressed as follows: the relevant representations for elementary particle physics are those of the form $\pi_{0} \circ \varrho$, where $\varrho \in \Delta_{t}$. A superselection sector may then be considered as the set of vector states of an irreducible representation of the form $\pi_{0} \circ \varrho$. This description does not do justice to the physical reasoning behind this basic assumption however we shall see how the set $\Delta_{t}$ arises naturally when analyzing the local cohomology. 
We let $\Sigma_{n}$ denote the set of $n$-simplexes ${ }^{2}$ in Minkowski space. To incorporate locality into the cohomology we need a good notion of a function on $\Sigma_{n}$ with values in In $\mathfrak{U}$, the group of inner automorphisms of $\mathfrak{A}$, or Mor $\mathfrak{A}$ which preserves localization regions. Inner automorphisms cannot, however, be localized as sharply as $n$-simplexes and to cope with this we allow for a certain uniform error specified by a double cone $\mathcal{O} \in \mathscr{K}_{0}$. We write $f \in C^{n}(\mathcal{O})$ if $f: \Sigma_{n} \rightarrow \operatorname{In} \mathfrak{A}$ and if $f(a)$ is localized in $\mathcal{O}+a=\left\{x+y: x \in \mathcal{O}, y=a\left(t^{0}, t^{1}, \ldots, t^{n}\right)\right\}$ for each $a \in \Sigma_{n}$. If $f \in \bigcup_{\mathcal{O} \in \mathscr{K}_{0}} C^{n}(\mathcal{O})$, we say that $f$ is a local function from $\Sigma_{n}$ to In $\mathfrak{A}$. We write $z \in Z^{1}(\mathcal{O})$ if $z \in C^{1}(\mathcal{O})$ and if $z$ is a 1-cocycle, i.e. if for every $c \in \Sigma_{2}$

$$
z\left(\partial_{0} c\right) z\left(\partial_{2} c\right)=z\left(\partial_{1} c\right)
$$

We write $Z^{1}=\bigcup_{\mathcal{O} \in \mathscr{K}_{0}} Z^{1}(\mathcal{O})$; an element of $Z^{1}$ will be called a local 1 -cocycle. A local 1-cocycle is the basic object of study in this paper and is supposed to represent the operation of transferring charge along a path as discussed in the introduction.

$$
\begin{aligned}
& \text { If } y \in C^{0}(\mathcal{O}) \text { and we define } \\
& d y(b)=y\left(\partial_{0} b\right) y\left(\partial_{1} b\right)^{-1}, \quad b \in \Sigma_{1}
\end{aligned}
$$

then $d y \in Z^{1}(\mathcal{O})$ and we say that $d y$ is a local 1-coboundary. More generally if $z^{\prime}$ and $z$ are local 1 -cocycles and if we can find a $y$ from some $C^{0}(\mathcal{O})$ such that

$$
z^{\prime}(b) y\left(\partial_{1} b\right)=y\left(\partial_{0} b\right) z(b), \quad b \in \Sigma_{1}
$$

then $z^{\prime}$ and $z$ will be said to be cohomologous. Cohomologous local 1-cocycle should be thought of as transferring the same charge.

As Minkowski space is contractible any 1-cocycle will be a 1-coboundary (Lemma A.2). Whilst this does not mean that any local 1-cocycle is a local 1-coboundary, it does imply that if $z \in Z^{1}$, then $z(b)$ depends only on $\partial_{0} b$ and $\partial_{1} b$. This has an important consequence for the localization properties of $z(b)$.

2.1. Lemma. If $z \in Z^{1}(\mathcal{O}), b \in \Sigma_{1}$, and $\mathcal{O}_{1} \subset\left(\mathcal{O}+\partial_{0} b\right)^{\prime} \cap\left(\mathcal{O}+\partial_{1} b\right)^{\prime}$ then

$$
z(b)(A)=A, \quad A \in \mathfrak{U}\left(\mathcal{O}_{1}\right) .
$$

Proof. Since $\left(\mathcal{O}_{1}-\mathcal{O}\right)^{\prime}$ is path-connected, we can find $b^{\prime} \in \Sigma_{1}$, with $\partial_{0} b^{\prime}=\partial_{0} b$, $\partial_{1} b^{\prime}=\partial_{1} b$ and $\mathcal{O}_{1} \subset\left(\mathcal{O}+b^{\prime}\right)^{\prime}$. Hence $z(b)(A)=z\left(b^{\prime}\right)(A)=A, A \in \mathfrak{A}\left(\mathcal{O}_{1}\right)$ as required.

We shall now describe a procedure which allows one to decide whether a given local 1-cocycle $z$ is a local 1-coboundary or not. The strategy is very simple; suppose $z=d y, y \in C^{\circ}(\mathcal{O})$ then we may compute $y$ as follows: given $a \in \Sigma_{0}, A \in \mathfrak{A}\left(\mathcal{O}_{1}\right)$, pick $b \in \Sigma_{1}$, with $\partial_{0} b=a$ and $\mathcal{O}+\partial_{1} b \subset \mathcal{O}_{1}^{\prime}$, then $y\left(\partial_{1} b\right)(A)=A$ and hence

$$
y(a)(A)=z(b)(A), \quad A \in \mathfrak{U}\left(\mathcal{O}_{1}\right) .
$$

We may use this procedure ${ }^{3}$ to define $y$ for any given $z \in Z^{1}$ and it turns out, in general, that $y$ takes values in $\Delta_{t}$ instead of In $\mathfrak{A}$.

2 The definition of an $n$-simplex and other elementary notions from cohomology may be found in the Appendix.

3 Compare the discussion of Lemma 3.1 in [5]. 
2.2. Theorem. If $z \in Z^{1}(\mathcal{O})$, there is a unique local function $y: \Sigma_{0} \rightarrow \Delta_{t}$ such that

$$
z(b) y\left(\partial_{1} b\right)=y\left(\partial_{0} b\right), \quad b \in \Sigma_{1} .
$$

Furthermore $y(a) \in \Delta_{t}(\mathcal{O}+a), a \in \Sigma_{0}$. If $z^{\prime}$ and $z$ are cohomologous so that there is a local function $w: \Sigma_{0} \rightarrow \operatorname{In} \mathfrak{A}$ such that

$$
z^{\prime}(b) w\left(\partial_{1} b\right)=w\left(\partial_{0} b\right) z(b), \quad b \in \Sigma_{1},
$$

then the associated functions, $y^{\prime}$ and $y$ are related by

$$
y^{\prime}(a)=w(a) y(a), \quad a \in \Sigma_{0} .
$$

Hence the equivalence class of $y(a)$ in $\Delta_{t}$ depends only on the cohomology class of $z$.

Proof. If $\mathrm{A} \in \mathfrak{A}\left(\mathcal{O}_{1}\right)$, we want to define $y(a)(A)$ as in (2.4), but must first show that this definition does not depend on the choice of $\partial_{1} b$. However if $c \in \Sigma_{2}, \partial_{0} \partial_{1} c=a$ and $\mathcal{O}_{1} \subset\left(\mathcal{O}+\partial_{1} \partial_{1} c\right)^{\prime} \cap\left(\mathcal{O}+\partial_{1} \partial_{0} c\right)^{\prime}$, then by Lemma 2.1, $z\left(\partial_{2} c\right)(A)=A$. The cocycle identity (2.2) now gives $z\left(\partial_{0} c\right)(A)=z\left(\partial_{1} c\right)(A)$, showing that $y(a)(A)$ in (2.4) is independent of $b$. It follows that $y(a)$ is a morphism on $\bigcup_{\mathcal{O} \in \mathscr{K}} \mathfrak{A}(\mathcal{O})$ and hence extends uniquely to a morphism of $\mathfrak{A}$. Lemma 2.1 implies that $y(a)$ is localized in $\mathcal{O}+a$ and (2.5) will show that $y(a) \in \Delta_{t}$. To prove (2.5), it suffices to prove it on each $\mathfrak{A}\left(\mathcal{O}_{1}\right)$ and this we do by choosing $c \in \Sigma_{2}$ with $\partial_{0} c=b$ and $\mathcal{O}_{1} \subset\left(\mathcal{O}+\partial_{1} \partial_{2} c\right)^{\prime}$ when (2.5) is a consequence of the cocycle identity (2.2). Similarly we prove (2.7) on each $\mathfrak{H}\left(\mathcal{O}_{1}\right)$ by first picking $\mathcal{O} \in \mathscr{K}_{0}$ sufficiently large so that $z, z^{\prime} \in Z^{1}(\mathcal{O})$ and $w \in C^{0}(\mathcal{O})$ and then picking $b \in \Sigma_{1}$ with $\partial_{0} b=a$ and $\mathcal{O}_{1} \subset\left(\mathcal{O}+\partial_{1} b\right)^{\prime}$ when (2.7) follows from (2.6).

There is one more step to take before we can claim that the local 1-cohomology and localized transportable morphisms give equivalent descriptions of superselection structure because we must show that every equivalence class in $\Delta_{t}$ corresponds to some cohomology class. This is achieved in the following theorem.

2.3. Theorem. Let $\mathcal{O} \in \mathscr{K}_{0}$ and $y: \Sigma_{0} \rightarrow \Delta_{t}$ be such that $y\left(a^{\prime}\right)$ is equivalent to $y(a) \in$ $\Delta_{t}(\mathcal{O}+a)$ for each $a, a^{\prime} \in \Sigma_{0}$. Then there is a $z \in Z^{1}(\mathcal{O})$ such that

$$
z(b) y\left(\partial_{1} b\right)=y\left(\partial_{0} b\right), \quad b \in \Sigma_{1} .
$$

Proof. If $b \in \Sigma_{1}, y\left(\partial_{1} b\right)$ and $y\left(\partial_{0} b\right)$ are equivalent by hypothesis so let $z(b)$ be any inner automorphism satisfying (2.8). If $\mathcal{O}_{1} \subset\left(\mathcal{O}+\partial_{1} b\right)^{\prime} \cap\left(\mathcal{O}+\partial_{0} b\right)^{\prime}, z(b)(A)=A$, for $A \in \mathfrak{U}\left(\mathcal{O}_{1}\right)$.

Hence we may find $z: \Sigma_{1} \rightarrow \operatorname{In} \mathfrak{A}$ satisfying (2.8) and it automatically has the correct localization properties although it is not necessarily a 1-cocycle. However if $h$ is any contracting homotopy [see Appendix, (A.7)] and we set

$$
z^{\prime}(b)=z\left(h \partial_{0} b\right) z\left(h \partial_{1} b\right)^{-1}
$$

then $z^{\prime}$ is a 1 -cocycle and still satisfies $(2.8)$. Hence $z^{\prime} \in Z^{1}(\mathcal{O})$ as required.

The reader may have noticed that the duality assumption has not been used in deriving the above results. However it is only under this assumption that the local 1-cocycles or localized transportable morphisms are necessarily associated with superselection structure. 
The local 1-cohomology remains unchanged if we were to use some other suitable set of simplexes on Minkowski space to define it. For example we could use $C^{\infty}$-simplexes or affine simplexes. One could also consider local 1-cocycles with values in $\mathscr{U}(\mathfrak{U})$, the unitary group of $\mathfrak{A}$, instead of $\operatorname{In} \mathfrak{A}$. We say that a unitary $u \in \mathscr{U}(\mathfrak{U})$ is localized in a compact set $F$ of Minkowski space if the corresponding inner automorphism $\sigma_{u}$ is localized there. The image of a local 1-cocycle in $\mathscr{U}(\mathfrak{U})$ under $\sigma$ is a local 1-cocycle in In $\mathfrak{A}$ to which Theorem (2.2) applies. Furthermore if $y: \Sigma_{0} \rightarrow \Delta_{t}$ is as in Theorem 2.3, then we may proceed as above to find a local 1 -cocycle $z$ with values in $\mathscr{U}(\mathfrak{U})$ satisfying

$$
\sigma_{z(b)} y\left(\partial_{1} b\right)=y\left(\partial_{0} b\right), \quad b \in \Sigma_{1} .
$$

Hence the resulting local 1-cohomology is again unchanged. This conclusion could be criticized on the grounds that the correct notion of a local function $z: \Sigma_{1} \rightarrow \mathscr{U}(\mathfrak{U})$ is that there is an $\mathcal{O} \in \mathscr{K}_{0}$ such that

$$
z(a) \in \bigvee_{s \in[0,1]} \mathfrak{A}(\mathcal{O}+a(s, 1-s)), \quad a \in \Sigma_{1} .
$$

However duality (1.5) implies that every local 1-cocycle is automatically a local function in this more restricted sense.

It is natural to ask how the analysis of superselection structure would look if presented in terms of local cohomology. The appropriate choice of coefficients for the cohomology here would be the algebraic system of localized morphisms and intertwiners. Such a development might lead to further insight into the structure, particularly as regards the geometry underlying the permutation symmetry (statistics) of a sector. However the investigation of higher cohomologies would seem to be a more urgent task.

\section{2-Dimensional Space-Time}

We turn now to consider the special case of a 2-dimensional space-time where the equivalence proved in Section 2 is no longer valid. There are two reasons for doing this; in the first place, a 2-dimensional space-time is, at present, a proving ground for field-theoretical models and it is as well to be prepared for structural phenomena peculiar to this situation. In the second place, there are physical phenomena associated with the $s$-dimensional local cohomology, where $s$ is the number of space-dimensions. In a 2-dimensional space-time such phenomena will appear in analyzing the local 1-cohomology. The motivating equation from the cohomology of differential forms is now the charge conservation equation

$$
\partial_{\mu} j^{\mu}(x)=0 \text {. }
$$

Using the metric tensor of Minkowski space, we get a dual tensor $j^{*}(x)$ whose exterior derivative vanishes, in other words a closed $s$-form. Now in quantum field theory, as is well-known, a conserved local current can be used to generate an internal symmetry of the system. The infinitesimal generator $\delta$ of such a symmetry is defined as follows: if $A \in \mathfrak{A}\left(\mathcal{O}_{R}\right)$, where $\mathcal{O}_{R}$ is the double cone whose base is the ball $x^{0}=0,|\boldsymbol{x}| \leqq R$,

$$
\delta(A)=i\left[j^{0}\left(g_{\varepsilon} f_{R^{\prime}}\right), A\right],
$$


where $R^{\prime}>R+\varepsilon, f_{R^{\prime}}$ and $g_{\varepsilon}$ are smooth functions of compact support such that $f_{R^{\prime}}(\boldsymbol{x})=1,|\boldsymbol{x}|<R^{\prime}, g_{\varepsilon}\left(x^{0}\right)=0$ if $\left|x^{0}\right|>\varepsilon$ and $\int g_{\varepsilon}\left(x^{0}\right) d x^{0}=1$, and

$$
j^{0}\left(g_{\varepsilon} f_{R^{\prime}}\right)=\int j^{0}\left(x^{0}, \boldsymbol{x}\right) g_{\varepsilon}\left(x^{0}\right) f_{R^{\prime}}(\boldsymbol{x}) d x^{0} d \boldsymbol{x} .
$$

If we look at this from the point of view of the dual tensor $j^{*}$, we would compute the generator as follows: let $h$ be a smooth approximation to the $\delta$-function, $\int h\left(x^{0}, \boldsymbol{x}\right) d x^{0} d \boldsymbol{x}=1$, with supp $h \subset \mathcal{O}_{\varepsilon}$ and let $j_{h}^{*}$ be the tensor obtained by smearing $j^{*}$ with $h$. We may consider the base of $\mathcal{O}_{R^{\prime}}$ as an $s$-simplex $b$ and then

$$
\delta(A)=i\left[j_{h}^{*}(b), A\right], \quad A \in \mathfrak{U}\left(\mathcal{O}_{R}\right)
$$

where $j_{h}^{*}(b)$ is the result of integrating the closed form $j_{h}^{*}$ over the $s$-simplex $b$. Of course it is perfectly possible that $\delta(A)=0$ for all $A$; this would be the case in particular if $j^{\mu}$ generates a gauge symmetry.

Now that we have described the construction of $\delta$ in cohomological terms, it is clear how one should proceed by analogy when the local cohomology takes its values in In $\mathfrak{A}$. If $z$ is a local s-cocycle and $A$ and $b$ are as in (3.3), we should be sometimes able to define a non-trivial internal symmetry $\zeta$ by setting

$$
\zeta(A)=z(b)(A), \quad A \in \mathfrak{U}\left(\mathcal{O}_{R}\right) .
$$

One may hope in this way to obtain discrete internal symmetries, whereas (3.1) will give only continuous internal symmetries.

In a 2-dimensional space-time if we could apply Lemma 2.1, we would be able to deduce that the internal symmetry $\zeta$ is trivial. However this lemma cannot be applied because if $\mathcal{O} \in \mathscr{K}, \mathcal{O}^{\prime}$ is no longer path-connected but decomposes into two components denoted by $\mathcal{O}^{r}$ and $\mathcal{O}^{l}$, the right and left spacelike complements of $\mathcal{O}$ respectively. $\mathcal{O}^{r}$ is defined by the convention that $(0, x) \in \mathcal{O}^{r}$ if $x$ is sufficiently positive. All we can say using the argument of Lemma 2.1 is:

3.1. Lemma. If $z \in Z^{1}(\mathcal{O}), b \in \Sigma_{1}$, and $i=r, l$, then

$$
z(b)(A)=A, \quad A \in \mathfrak{U}\left(\mathcal{O}_{1}\right)
$$

whenever $\mathcal{O}_{1} \subset\left(\mathcal{O}+\partial_{0} b\right)^{i} \cap\left(\mathcal{O}+\partial_{1} b\right)^{i}$.

In place of Theorem 2.2 we have

3.2. Proposition. If $z \in Z^{1}(\mathcal{O})$ and $i=r$ or $l$ then there is a unique $y^{i}: \Sigma_{0} \rightarrow \operatorname{Mor} \mathfrak{A}$ such that

$$
z(b) y^{i}\left(\partial_{1} b\right)=y^{i}\left(\partial_{0} b\right), \quad b \in \Sigma_{1}
$$

and if $\mathcal{O}_{1} \subset(\mathcal{O}+a)^{i}$

$$
y^{i}(a)(A)=A, \quad A \in \mathfrak{U}\left(\mathcal{O}_{1}\right) .
$$

If $z^{\prime}$ and $z$ are cohomologous, so that there is a local function $w: \Sigma_{0} \rightarrow \operatorname{In} \mathfrak{A}$ with

$$
z^{\prime}(b) w\left(\partial_{1} b\right)=w\left(\partial_{0} b\right) z(b), \quad b \in \Sigma_{1}
$$

then the associated functions $y^{\prime i}$ and $y^{i}$ are related by

$$
y^{\prime i}(a)=w(a) y^{i}(a), \quad a \in \Sigma_{0} .
$$


Thus one may associate with a local 1-cocycle morphisms which are either left localized or right localized. Unfortunately there seems, in general, to be no information either on the relationship between $y^{l}$ and $y^{r}$ or on the way $y^{l}$ acts on the spacelike right and $y^{r}$ on the spacelike left. The best we can do is to impose an additonal locality requirement on local 1-cocycles which allows more precise results in the direction anticipated by the discussion at the beginning of the section.

We write $z \in \tilde{Z}^{1}(\mathcal{O})$ if $z \in Z^{1}(\mathcal{O})$ and if for any $c \in \Sigma_{2}$, and $\mathcal{O}_{1} \in \mathscr{K}, \mathcal{O}_{1} \subset\left(\mathcal{O}+\partial_{1} \partial_{1} c\right)^{\prime} \cap$ $\left(\mathcal{O}+\partial_{0} c\right)^{\prime}$.

$$
z\left(\partial_{2} c\right)^{-1} z\left(\partial_{0} c\right) z\left(\partial_{2} c\right)(A)=A, \quad A \in \mathfrak{A}\left(\mathcal{O}_{1}\right) .
$$

In some ways this additional requirement may be looked on as a penalty for indulging in non-Abelian cohomology. Certainly if $z$ takes its values in some Abelian subgroup of In $\mathfrak{A}$ then (3.8) is a consequence of the locality of $z$. In more than two space-time dimensions, Lemma 2.1 implies that any local cocycle satisfies (3.8).

3.3. Theorem. Let $z \in \tilde{Z}^{1}(\mathcal{O})$ and let $i, j, k$ denote $l$ or $r$ then there are unique automorphisms $\zeta_{i j}$ such that if $\mathcal{O}_{1} \in \mathscr{K}$ and $b \in \Sigma_{1}$, with $\partial_{0} b+\mathcal{O} \subset \mathcal{O}_{1}^{i}, \partial_{1} b+\mathcal{O} \subset \mathcal{O}_{1}^{j}$ then

$$
\zeta_{i j}(A)=z(b)(A), \quad A \in \mathfrak{A}\left(\mathcal{O}_{1}\right) .
$$

Furthermore $\zeta_{i i}$ is the identity and

$$
\begin{aligned}
\zeta_{i j} \zeta_{j k} & =\zeta_{i k}, \\
y^{k}(a) \zeta_{j k} & =y^{j}(a), \quad a \in \Sigma_{0} .
\end{aligned}
$$

If $q$ is a real number $>2$ then

$$
\zeta_{i j}(A) \in \mathfrak{A}\left(\mathcal{O}_{1}+q \mathcal{O}\right), \quad A \in \mathfrak{A}\left(\mathcal{O}_{1}\right) .
$$

If $z^{\prime}$ is cohomologous to $z$, then $z^{\prime} \in \tilde{Z}^{1}=\bigcup_{\mathcal{O} \in \mathscr{K}_{0}} \tilde{Z}^{1}(\mathcal{O})$ and $z^{\prime}$ gives rise to the same automorphisms $\zeta_{i j}$.

Proof. The uniqueness of the automorphisms follows from (3.9); what really has to be checked is that the right hand side of (3.9) is independent of $b$ under the stated conditions. If $i=j$ this follows from Lemma 3.1 which states that $\zeta_{i i}$ is the identity. If $i \neq j$ then $\mathcal{O}_{1} \subset\left(\partial_{1} b+\mathcal{O}\right)^{i}$ and (3.5) and (3.6) show that $z(b)(A)=y^{i}\left(\partial_{0} b\right)(A)$ so that $z(b)(A)$ can depend only on $\partial_{0} b$. Given $c \in \Sigma_{2}$ with $\mathcal{O}+\partial_{0} c \subset \mathcal{O}_{1}^{i}$ and $\mathcal{O}+\partial_{1} \partial_{1} c \subset \mathcal{O}_{1}^{j}$, the cocycle identity implies

$$
z\left(\partial_{1} c\right)(A)=z\left(\partial_{0} c\right) z\left(\partial_{2} c\right)(A)=z\left(\partial_{2} c\right)\left(z\left(\partial_{2} c\right)^{-1} z\left(\partial_{0} c\right) z\left(\partial_{2} c\right)\right)(A) .
$$

Applying (3.8) we deduce that $z\left(\partial_{1} c\right)(A)=z\left(\partial_{2} c\right)(A)$ and this is enough to show that $z(b)(A)$ in (3.9) is independent of $\partial_{0} b$. As usual, this independence implies that $\zeta_{i j}$ is a morphism. Now by duality we know that $z(b)$ is induced by a unitary from $\mathfrak{A}\left(\mathcal{O}_{2}\right)$ where $\mathcal{O}_{2}$ is the smallest double cone containing $\mathcal{O}+\partial_{1} b$ and $\mathcal{O}+\partial_{0} b$. However we may pick $b \in \Sigma_{1}$ such that $\mathcal{O}_{2}=q \mathcal{O}+\mathcal{O}_{1}$ and $\zeta_{i j}(A)=z(b)(A), A \in \mathfrak{A}\left(\mathscr{O}_{1}\right)$. This proves (3.12). 
Now pick $c \in \Sigma_{2}$ such that $\mathcal{O}+\partial_{1} \partial_{2} c \subset\left(\mathcal{O}_{1}+q \mathcal{O}\right)^{k}, \mathcal{O}+\partial_{0} \partial_{2} c \subset\left(\mathcal{O}_{1}+q \mathcal{O}\right)^{j}$ and $\mathcal{O}+\partial_{0} \partial_{1} c \subset\left(\mathcal{O}_{1}+q \mathcal{O}\right)^{i}$, evaluate the 2-cocycle identity on $A \in \mathfrak{A}\left(\mathcal{O}_{1}\right)$ using (3.12) and we deduce (3.10). This also shows that $\zeta_{i j}$ is an automorphism. If we leave $\partial_{1} \partial_{2} c$ and $\partial_{0} \partial_{2} c$ as above and pick $\partial_{0} \partial_{1} c=a$ then the cocycle identity implies (3.11).

If $z^{\prime}$ is cohomologous to $z$, there is a local function $w: \Sigma_{0} \rightarrow \operatorname{In} \mathfrak{A}$ so that (2.6) holds. We may suppose without loss of generality that $\mathscr{O} \in \mathscr{K}_{0}$ has been chosen so that $z^{\prime} \in Z^{1}(\mathcal{O})$ and $w \in C^{0}(\mathcal{O})$. Now pick $b \in \Sigma_{1}$ such that $\mathcal{O}+\partial_{1} b C\left(\mathcal{O}_{1}+q \mathcal{O}\right)^{j}$ and $\mathcal{O}+\partial_{0} b \subset\left(\mathcal{O}_{1}+q \mathcal{O}\right)^{i}$, apply (2.6) to $A \in \mathfrak{U}\left(\mathcal{O}_{1}\right)$, and we get using (3.12),

$$
z^{\prime}(b)(A)=\zeta_{i j}(A), \quad A \in \mathfrak{U}\left(\mathcal{O}_{1}\right) .
$$

If we now return to (3.8), we see that this suffices to show that $z^{\prime} \in \tilde{Z}^{1}(q \mathcal{O})$.

Actually duality and covariance of the vacuum are enough to show that we may take $q=2$ in (3.12). It should, however, be noted that (3.12) does not imply that $\zeta_{l r}$ is an internal symmetry; $\zeta_{l r}$ might implement the space-time translation through some $x \in \mathcal{O}$ for example. Indeed this situation is not so unlikely if we realize that $j^{\mu}$ in (3.1) need not transform like a vector under the Poincare group but might be $\theta^{\mu 1}$, say, where $\theta^{\mu \nu}$ is the energy-momentum density. On the other hand, if the cohomology class of $z$ contains elements of $\tilde{Z}^{1}(\mathcal{O})$ for arbitrarily small $\mathcal{O} \in \mathscr{K}_{0}$, then $\zeta_{\text {lr }}$ will be an internal symmetry.

Now that we know that $y^{r}(a)$ behaves like $\zeta_{r l}$ on the spacelike left, we have enough information to give an analogue of Theorem 2.3.

3.4. Theorem. Let $\mathcal{O} \in \mathscr{K}_{0}$ and let $\zeta$ be an automorphism of $\mathfrak{U}$ such that

$$
\zeta^{-1}\left(\mathfrak{U}\left(\mathcal{O}_{1}\right)\right) \subset \mathfrak{U}\left(\mathcal{O}_{1}+\mathcal{O}\right), \quad \mathcal{O}_{1} \in \mathscr{K} .
$$

Let $y^{r}: \Sigma_{0} \rightarrow \operatorname{Mor} \mathfrak{A}$ be such that $y^{r}(a)$ is equivalent to $y^{r}\left(a^{\prime}\right)$ for $a, a^{\prime} \in \Sigma_{0}$ and

$$
\begin{array}{ll}
y^{r}(a)(A)=A, & A \in \mathfrak{U}\left(\mathcal{O}^{r}+a\right), \\
y^{r}(a)(A)=\zeta(A), & A \in \mathfrak{U}\left(\mathcal{O}^{l}+a\right) .
\end{array}
$$

Then there is a local 1-cocycle $z \in \tilde{Z}^{1}(q \mathcal{O}), q>2$ with

$$
z(b) y^{r}\left(\partial_{1} b\right)=y^{r}\left(\partial_{0} b\right), \quad b \in \Sigma_{1} .
$$

$\zeta$ is the automorphism $\zeta_{r l}$ associated with $z$ in Theorem 3.3.

Proof. (3.15) for $z \in \tilde{Z}^{1}(q \mathcal{O})$ implies that $\zeta$ is the automorphism $\zeta_{r l}$ associated with $z$ in Theorem 3.3. The remainder of the theorem will follow as in the proof of Theorem 2.3 provided we can show that any inner automorphism satisfying (3.15) automatically has the correct localization properties. However if $\mathcal{O}_{1} \subset(q \mathcal{O}+b)^{\prime}$ then either $\mathcal{O}_{1} \subset\left(q \mathcal{O}+\partial_{0} b\right)^{l} \cap\left(q \mathcal{O}+\partial_{1} b\right)^{l}$ or $\mathcal{O}_{1} \subset\left(q \mathcal{O}+\partial_{1} b\right)^{r} \cap\left(q \mathcal{O}+\partial_{0} b\right)^{r}$. We deduce that $z(b)(A)=A, A \in \mathfrak{U}\left(\mathcal{O}_{1}\right)$ by applying (3.15) to $\zeta^{-1}(A) \in \mathfrak{U}\left(\mathcal{O}_{1}+\mathcal{O}\right)$ in the former case and directly to $A$ in the latter case.

This concludes our discussion of the local 1-cohomology in a 2-dimensional space-time. We have, at least for local cocycles in $\tilde{Z}^{1}$, reduced the study of this cohomology to the study of transportable morphisms which are, say, right localized and behave on the left like some almost local automorphism. How much can be said about such morphisms is not yet clear and it is worth noting 
that there has been, as yet, no systematic study even of transportable localized morphisms in a 2-dimensional space-time. One knows that the basic Lemmas 2.4 and 4.1 of [5] fail in this case. The proof breaks down because $\mathcal{O}^{\prime}$ is no longer path-connected. The physical reason why the results must fail is that even if $\delta=0$ in (3.2) we might be generating a non-trivial gauge symmetry and this influences the commutation relations of the associated unobservable fields.

Of course any theoretical analysis of what might happen must be backed up by models showing what does happen so as to minimize and localize the speculative content. Such models do exist in the literature and deserve a more detailed comment than that given here. The earliest such model is the treatment given by Streater and Wilde [8] of the free Skyrme model. From the point of view of the local cohomology it is a very natural model to think of: there is no free scalar field of mass zero in two dimensions so the conserved current which ought to be its derivative should, and does, generate non-trivial local 1-cocycles. This work was extended by Bonnard and Streater [9] to the massive free field with $U(1)$ or $O(2)$ symmetry. However, undoubtedly the most interesting results to date have been those obtained by Fröhlich [10]. They show how the local 1-cohomology in two space-time dimensions can generate the soliton sectors for interacting models with spontaneously broken internal symmetry such as the quantum "sine-Gordon" model. This is best seen by comparing Equation (77) of [10] with Equation (3.14) above.

\section{Appendix}

We gather together here for the reader's convenience a few basic definitions and elementary facts relating to cohomology. The reader wishing more detailed information might consult, for example [11]. The standard $n$-simplex, denoted by $\Delta^{n}$ is $\left\{\left(t^{0}, t^{1}, \ldots, t^{n}\right) \in \mathbb{R}^{n+1}: t^{i} \geqq 0\right.$ and $\left.\sum_{i=0}^{n} t^{i}=1\right\}$. There are affine mappings, $d_{i}: \Delta^{n} \rightarrow \Delta^{n+1}, i=0,1,2, \ldots, n+1$ and $s_{j}: \Delta^{n} \rightarrow \Delta^{n-1}, j=0,1,2, \ldots, n-1$ defined by

$$
\begin{aligned}
& d_{i}\left(t^{0}, t^{1}, \ldots, t^{n}\right)=\left(t^{0}, t^{1}, \ldots, t^{i-1}, 0, t^{i}, \ldots, t^{n}\right), \\
& s_{j}\left(t^{0}, t^{1}, \ldots, t^{n}\right)=\left(t^{0}, t^{1}, \ldots, t^{j-1}, t^{j}+t^{j+1}, t^{j+2}, \ldots, t^{n}\right) .
\end{aligned}
$$

Any orientation preserving affine mapping from $\Delta^{m}$ to $\Delta^{n}$ is a composition of mappings of this sort. The following rules may be used to manipulate composite mappings

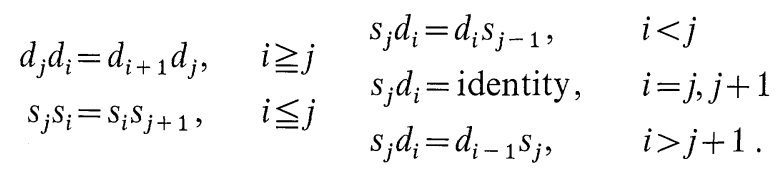

If $X$ is a topological space, an $n$-simplex of $X$ is a continuous mapping $a: \Delta^{n} \rightarrow X$. The set of $n$-simplexes of $X$ is denoted by $\Sigma_{n}(X)$. The mappings $d_{i}$ and $s_{j}$ induce operations on the simplexes of $X:$ if $a \in \Sigma_{n}(X)$,

$$
\begin{aligned}
\partial_{i} a=a d_{i}, & i=0,1,2, \ldots, n \\
\sigma_{j} a=a s_{j}, & j=0,1,2, \ldots, n .
\end{aligned}
$$


These operations are called the boundary and degeneracy operations on the simplexes of $X$ respectively, and (A.2) gives

$$
\begin{array}{llll}
\partial_{i} \partial_{j}=\partial_{j} \partial_{i+1}, & i \geqq j & \partial_{i} \sigma_{j}=\sigma_{j-1} \partial_{i}, & i<j \\
\sigma_{i} \sigma_{j}=\sigma_{j+1} \sigma_{i}, & i \leqq j & \partial_{i} \sigma_{j}=\text { identity, } & i=j, j+1 \\
& \partial_{i} \sigma_{j}=\sigma_{j} \partial_{i-1}, & i>j+1 .
\end{array}
$$

A 0 -simplex of $X$ may be considered as a point of $X$ and a 1 -simplex is a path in $X$. If $a \in \Sigma_{n}(X), n>0$, then we define $\bar{a} \in \Sigma_{n}(X)$, the oppositely oriented simplex by

$$
\bar{a}\left(t^{0}, t^{1}, \ldots, t^{n}\right)=a\left(t^{n}, t^{n-1}, \ldots, t^{0}\right) .
$$

Suppose $X$ in a convex set and let $x_{0} \in X$ then we can define a mapping $h: \Sigma_{n}(X) \rightarrow \Sigma_{n+1}(X)$ as follows

$$
\begin{aligned}
h(a)\left(t^{0}, t^{1}, \ldots, t^{n+1}\right) & =t^{0} x_{0}+\left(1-t^{0}\right) a\left(\tau t^{1}, \tau t^{2}, \ldots, \tau t^{n}\right), \quad t^{0} \neq 1 \\
& =x_{0}, \quad t^{0}=1 .
\end{aligned}
$$

Here we have written $\tau=\left(1-t^{0}\right)^{-1}$. The geometric interpretation of $h(a)$ is that it is the $n+1$-simplex constructed by joining each vertex of the $n$-simplex $a$ by a straight line to the point $x_{0}$. We have

$$
\partial_{0} h(a)=a, \quad \partial_{i} h(a)=h\left(\partial_{i-1} a\right), \quad i>0
$$

except that if $a \in \Sigma_{0}(X)$ then $\partial_{1} h(a)=x_{0}$. A mapping satisfying (A.7) is called a contracting homotopy.

Cohomology usually means Abelian cohomology; however we only need the formal definition of the 1-cohomology and there is no difficulty in defining the 1 -cohomology with values in some non-Abelian group $G$.

A 1-cocycle with values in a group $G$ is a function $z: \Sigma_{1}(X) \rightarrow G$ such that

$$
z\left(\partial_{0} c\right) z\left(\partial_{2} c\right)=z\left(\partial_{1} c\right), \quad c \in \Sigma_{2}(X) .
$$

A function $z: \Sigma_{1}(X) \rightarrow G$ is a 1-coboundary if there is a function $w: \Sigma_{0}(X) \rightarrow G$ such that

$$
z(b)=w\left(\partial_{0} b\right) w\left(\partial_{1} b\right)^{-1}, \quad b \in \Sigma_{1}(X) .
$$

Every 1-coboundary is a 1-cocycle. Two 1-cocycles are said to be cohomologous if there is a function $w: \Sigma_{0}(X) \rightarrow G$ such that

$$
z^{\prime}(b) w\left(\partial_{1} b\right)=w\left(\partial_{0} b\right) z(b), \quad b \in \Sigma_{1}(X) .
$$

This defines an equivalence relation on the set of 1-cocycles and the corresponding equivalence classes are called cohomology classes.

\section{A.1. Lemma. Let $z$ be a 1-cocycle then}

$$
\begin{aligned}
z\left(\sigma_{0} a\right) & =1, & & a \in \Sigma_{0}(X), \\
z(b) & =z(\bar{b})^{-1}, & & b \in \Sigma_{1}(X) .
\end{aligned}
$$


Proof. Given $a \in \Sigma_{0}(X)$, apply (A.8) to $\sigma_{0} \sigma_{0} a \in \Sigma_{2}(X)$ and we get using (A.4), $z\left(\sigma_{0} a\right) z\left(\sigma_{0} a\right)=z\left(\sigma_{0} a\right)$, which implies $z\left(\sigma_{0} a\right)=1$. Given $b \in \Sigma_{1}(X)$, define $c \in \Sigma_{2}(X)$ by $c\left(t^{0}, t^{1}, t^{2}\right)=b\left(t^{0}+t^{2}, t^{1}\right)$ and we get applying (A.8) $z(\bar{b}) z(b)=z\left(\sigma_{0} \partial_{1} b\right)=1$.

A.2. Lemma. If $X$ is a convex set, or, more generally, if we have a contracting homotopy $h: \Sigma_{n}(X) \rightarrow \Sigma_{n+1}(X)$ then every 1-cocycle is a 1-coboundary.

Proof. Let $z$ be a 1-cocycle. Given $a \in \Sigma_{0}(X)$ define $w(a)=z(h(a))$. Given $b \in \Sigma_{1}(X)$, apply (A.8) to $h(b) \in \Sigma_{2}(X)$ and we get using (A.7), $z(b) w\left(\partial_{1} b\right)=w\left(\partial_{0} b\right)$ showing that $z$ is a 1 -coboundary.

Acknowledgements. R. Haag has stressed to me on several occasions over the past years the importance of locality as the novel ingredient of a cohomology of differential forms in quantum field theory, citing quantum electrodynamics as an example. His remarks eventually fell on fruitful ground. D. Buchholz has suggested to me that looking at path-dependent unitary operators might be a good way of trying to exploit these ideas in an algebraic setting. From here it is but a short step to realize that the resulting 1 -cohomology is just the existing theory of supersection sectors.

\section{References}

1. Haag, R., Kastler, D. : An algebraic approach to quantum field theory. J. Math. Phys. 5, 848 - 861 (1964)

2. Borchers, H.J.: Local rings and the connection of spin with statistics. Commun. math. Phys. 1, 281-307 (1965)

3. Doplicher, S., Haag, R., Roberts, J.E.: Fields, observables and gauge transformations. I. Commun. math. Phys. 13, 1-23 (1969)

4. Doplicher, S., Haag, R., Roberts, J. E.: Fields, observables and gauge transformation. II. Commun. math. Phys. 15, 173-200 (1969)

5. Doplicher, S., Haag, R., Roberts, J.E.: Local observables and particle statistics. I. Commun. math. Phys. 23, $199-230$ (1971)

6. Doplicher,S., Haag, R., Roberts, J.E.: Local observables and particle statistics. II. Commun. math. Phys. 35, 49-85 (1974)

7. Pohlmeyer, K.: The equation curl $W_{\mu}(x)=0$ in quantum field theory. Commun. math. Phys. 25, $73-86$ (1972)

8. Streater, R.F., Wilde,I.F.: Fermion states of a boson field. Nuclear Phys. B 24, 561-575 (1970)

9. Streater,R.F.: Local gauge models predicting their own superselection rules. Report to the Moscow conference on mathematical problems of quantum field theory and quantum statistical mechanics 1972. Helv. Phys. Acta 49, 259-267 (1976)

10. Fröhlich, J.: New superselection sectors ("soliton-states") in two-dimensional bose quantum field models. Commun. math. Phys. 47, 269-310 (1976)

11. Mac Lane, S.: Homology. Berlin, Heidelberg, New York: Springer 1963

Communicated by R. Haag

Received April 5, 1976 
